\title{
Comparison of urine albumin creatinine ratio with the pediatric index of mortality 2 score for prediction of pediatric intensive care unit outcomes
}

\author{
Shifa Nismath ${ }^{1}$-Suchetha S. Rao ${ }^{1}$ (1) B. S. Baliga ${ }^{1} \cdot$ Vaman Kulkarni $^{2} \cdot$ Gayatri M. Rao $^{3}$
}

Received: 19 July 2021 / Accepted: 25 August 2021 / Published online: 9 September 2021

(C) The Author(s) 2021

\begin{abstract}
Background Predicting morbidity and mortality in a pediatric intensive care unit (PICU) is of extreme importance to make precise decisions for better outcomes.

Aim We compared the urine albumin creatinine ratio (ACR) with the established PICU score, pediatric index of mortality 2 (PIM 2) for predicting PICU outcomes.

Methods This cross-sectional study enrolled 67 patients admitted to PICU with systemic inflammatory response syndrome. Urine ACR was estimated on admission, and PIM 2 score was calculated. ACR was compared with PIM 2 for PICU outcome measures: the need for inotropes, development of multiple organ dysfunction syndrome (MODS), duration of PICU stay, and survival.

Results Microalbuminuria was found in $77.6 \%$ of patients with a median ACR of $80 \mathrm{mg} / \mathrm{g}$. ACR showed a significant association with the need for inotropes $(p<0.001)$, MODS $(p=0.001)$, and significant correlation to PICU stay $(p 0.001$, rho $=0.361)$. The area under the receiver operating characteristic curve for ACR $(0.798)$ was comparable to that of PIM 2 (0.896). The cutoff value of ACR derived to predict mortality was $110 \mathrm{mg} / \mathrm{g}$. The study subjects were divided into 2 groups: below cutoff and above the cutoff. Outcome variables, inotrope use, MODS, mortality, and PICU stay compared between these subgroups, were statistically significant.

Conclusion ACR is a good predictor of PICU outcomes and is comparable to PIM 2 for mortality prediction.
\end{abstract}

Keywords Critical illness $\cdot$ Microalbuminuria $\cdot$ Mortality $\cdot$ Multiple organ dysfunction syndrome $\cdot$ Prognosis

$\begin{array}{ll}\text { Abbreviations } \\ \text { ACR } & \text { Albumin creatinine ratio } \\ \text { AUC } & \text { Area under the curve } \\ \text { INR } & \text { International normalized ratio } \\ \text { IQR } & \text { Interquartile range } \\ \text { MODS } & \text { Multiple organ dysfunction syndrome } \\ \text { Pa02 } & \text { Partial pressure of arterial oxygen }\end{array}$

Suchetha S. Rao

suchetha.rao@manipal.edu

1 Department of Pediatrics, Kasturba Medical College, Mangalore, Manipal Academy of Higher Education, Manipal, India

2 Department of Community Medicine, Kasturba Medical College, Mangalore, Manipal Academy of Higher Education, Manipal, India

3 Department of Biochemistry, Kasturba Medical College, Mangalore, Manipal Academy of Higher Education, Manipal, India

\author{
PELOD Pediatric logistic organ dysfunction sore \\ PICU Pediatric intensive care unit \\ PIM 2 Pediatric index of mortality 2 \\ PRISM Pediatric risk of mortality \\ ROC Receiver operating characteristic \\ SGOT Serum glutamic oxaloacetic transaminase
}

\section{Introduction}

Predicting morbidity and mortality in pediatric intensive care units is of extreme importance and can be challenging. Appropriate prediction helps to make the right decisions and thereby improve outcomes [1]. Many clinical scores like pediatric index of mortality 2 (PIM 2), pediatric risk of mortality (PRISM) score, and pediatric logistic organ dysfunction (PELOD) score are used to predict PICU outcomes $[1,2]$. These scores have been validated in ICU settings 
of developed countries [3, 4]. Calculation of these scores requires many variables and internet facilities.

In critically ill patients, inflammatory response leads to endothelial membrane damage, a subsequent increase in capillary permeability results in transient albuminuria. The extent of albuminuria is variable, and most instances are not measured by urine dipstick protein estimation, hence known as microalbuminuria. The microalbuminuria can be measured by spot urine albumin creatinine ratio (ACR) [5]. The ACR is a simple, easy, and non-invasive measure that can be a good predictor of PICU outcome $[6,7]$.

We aimed to compare the ACR with the established PICU scoring system, PIM 2 as a predictor of PICU outcome. We decided to opt for PIM 2 as it is taken at $1 \mathrm{~h}$ from admission, which eliminates treatment-related changes in the scoring [4].

\section{Method}

A cross-sectional, analytical study was conducted from January 2016 to September 2016, in tertiary care teaching hospitals affiliated to Kasturba Medical College Mangalore, Manipal Academy of Higher Education. Institutional ethics committee permission was obtained before the start of the study. Permission was obtained from the Medical Superintendent of the hospitals.

A sample size of 67 was calculated with a $95 \%$ confidence interval, $10 \%$ margin of error, and using data from a previous study by Basu et al. which showed a prevalence of $78 \%$ of microalbuminuria in the first $6 \mathrm{~h}$ of admission [8].

Patients with systemic inflammatory response syndrome admitted to PICU were included [9]. Patients with chronic renal disease, acute kidney injury, urinary tract infections, nephrotic syndrome, acute glomerulonephritis, and nephrotoxic drugs were excluded. A patient information sheet was given; the purpose of the study was explained, and written informed consent was obtained from parents.

Blood investigations including those required to calculate the PIM 2 score were sent on admission, and urine sample was collected within $1 \mathrm{~h}$ of admission by direct catch or by catheterization for estimation of albumin creatinine ratio. Variables used for PIM 2 score calculation were elective admission to the PICU, recovery post-procedure, admitted following cardiac bypass, high-risk diagnosis, low-risk diagnosis, no response of pupils to bright light, mechanical ventilation if required anytime during the first hour in PICU, systolic blood pressure $\mathrm{mm} \mathrm{Hg}$, base excess, and partial pressure of arterial oxygen $\left(\mathrm{PaO}_{2}\right)$. PIM 2 score was calculated using an online calculator, and a predicted death rate was found [10].

Urine creatinine was estimated using the Jaffe method, and urinary albumin was estimated using a kit based on the immunoturbid metric method, using semi auto analyzer. Urine ACR was calculated. Urine ACR ratio $30-300 \mathrm{mg} / \mathrm{g}$ creatinine suggested microalbuminuria [5]. Patients were followed up subsequently until their PICU stay about the need for inotropes, development of multiple organ dysfunction syndrome (MODS), recovery, or mortality. The total duration of PICU stay was noted.

\section{Statistical analysis}

The collected data was coded and entered on the Statistical Package for IBM (SPSS) Statistics for Windows version 25.0, Armonk, NY, IBM Corp. Descriptive data were expressed as percentages, mean, standard deviation, or median. Mann-Whitney $U$ test was used to compare parameters between survivors and non-survivors. A $p<0.05$ was considered statistically significant. The receiver operating characteristic (ROC) curve was constructed, and the area under the curve was calculated.

\section{Results}

A total of 67 patients admitted to PICU were enrolled in the study. The median age of the participants was 2 years (IQR $0.41-6$ ). The study comprised $52.2 \%$ males and $47.8 \%$ females. Most of the cases belonged to respiratory illness 31 (46.3\%) like pneumonia and acute respiratory distress syndrome. Microalbuminuria was found in 52 patients (77.6\%); median ACR was 80 (IQR 36. 31-236.14). Median PIM 2 score was 1.8 (IQR 1.2-2.2) Median PICU stay was 4 days (IQR 3-6). Six (8.9\%) deaths were observed in PICU. A comparison of parameters between survivors and non-survivors is depicted in Table 1.

ACR had a significant correlation to PICU stay ( $p$ 0.001 , rho $=0.361)$. Among the study subjects, $14(20.9 \%)$ required inotropes and 15 (22.4\%) developed MODS. ACR showed a statistically significant association with the need for inotropes $(p<0.001)$ and the development of MODS $(p=0.001)$. ROC curve for ACR and need of inotrope and MODS are shown in Fig. 1a, b, respectively. AUC for the need of inotrope was 0.819 , and MODS was 0.781 , respectively (Fig. 1a, b).

As per the ROC curve, AUC derived for mortality for ACR was 0.798, and AUC for mortality for PIM 2 was 0.896. The AUC for ACR was comparable to the standard clinical mortality score PIM 2 (Fig. 2). The cutoff value of ACR derived to predict mortality was $110 \mathrm{mg} / \mathrm{g}$ and had a sensitivity of and specificity of $83.3 \%$ and $41.1 \%$. Based on this cutoff value, the study subjects were divided into 2 groups: below cutoff $(\leq 110 \mathrm{mg} / \mathrm{g})$ and above cutoff $(>110 \mathrm{mg} / \mathrm{g})$. Outcome variables such as the need for 
Table 1 Comparison of parameters between survivors and non-survivors

\begin{tabular}{llll}
\hline Variables & $\begin{array}{l}\text { Non-survivors, } n=6 \\
\text { Median (IQR) }\end{array}$ & $\begin{array}{l}\text { Survivors, } n=61 \\
\text { Median (IQR) }\end{array}$ & $p$ \\
\hline Hemoglobin (gm/dl) & $8.60(7.22-11.05)$ & $10.00(9.30-11.70)$ & 0.109 \\
Total counts (cells/cumm) & $25,150(12,000-34,375)$ & $12,700(6550-17,000)$ & 0.025 \\
Absolute neutrophil count & $11,936(10,960-15,864)$ & $5600(2514-11,377.5)$ & 0.022 \\
$\quad$ cells/cumm) & $224,500(162,000-393,250)$ & $334,000(180,000-486,000)$ & 0.345 \\
Platelet (cells/cumm) & $0.45(0.30-1.02)$ & $0.30(0.30-0.50)$ & 0.121 \\
Creatinine (mg/dl) & $67.50(39.00-99.00)$ & $33(22-47)$ & 0.055 \\
SGOT (U/l) & $19.60(15.85-33.97)$ & $15.50(14.80-16.00)$ & 0.016 \\
Prothrombin time (s) & $1.47(1.20-2.96)$ & $1.20(1.09-1.40)$ & 0.161 \\
INR & $0.59(0.33-1.01)$ & $0.32(0.21-0.56)$ & 0.227 \\
Total bilirubin (mg/dl) & $9.05(7.82-9.40)$ & $9.00(8.80-9.45)$ & 0.815 \\
Calcium (mg/dl) & $4.36(3.94-4.84)$ & $4.60(4.20-5.07)$ & 0.339 \\
Potassium (mmol/l) & $11.85(6.80-15.65)$ & $16.80(14.35-19.20)$ & 0.017 \\
Bicarbonate (mmol/l) & $86.90(51.57-137.50)$ & $98(90-110.5)$ & 0.402 \\
PaO (mmHg) & $-10.90(-26.10--2.75)$ & $-4.00(-5.50--2.00)$ & 0.135 \\
Base excess (mmol/l) & $361.50(191.00-664.25)$ & $70.36(32.80-196.00)$ & 0.017 \\
ACR (mg/g) & $23.20(2.55-55.85)$ & $1.70(1.20-2.10)$ & 0.01 \\
PIM2 & $6(3-17.25)$ & $2(2-5)$ & 0.012 \\
PICU days & & & \\
\hline
\end{tabular}

$I Q R$ interquartile range, $S G O T$ serum glutamic oxaloacetic transaminase, $\mathrm{PaO}_{2}$ partial pressure of oxygen, $I N R$ international normalized ratio, ACR albumin creatinine ratio, PIM2 pediatric index of mortality 2, $P I C U$ pediatric intensive care unit inotropes, development of MODS, mortality, and median PICU stay days compared between these subgroups were statistically significant (Table 2).

\section{Discussion}

Systemic inflammation is associated with increased permeability to plasma proteins which usually returns to normal within $48 \mathrm{~h}$ with the improvement in the disease process. As renal tubules have limited capacity to handle albumin reabsorption, increased capillary permeability leads to increased urinary albumin excretion [11].

In the present study, ACR was calculated within $1 \mathrm{~h}$ of admission, which showed the presence of MA in $77.6 \%$ of patients. A study by Ayse et al. reported the prevalence of MA in $64 \%$ of critically ill children. They found that MA was much higher in children with sepsis. They also concluded that ACR at admission as well as at $24 \mathrm{~h}$ had a good correlation with clinical scores (PELOD, PRISM, and PIM 2) for mortality prediction. ACR levels were associated with the duration of mechanical ventilation, the need for inotropic support, and the extent of MODS [5]. A study by Basu et al. found that $78 \%$ admitted to ICU had microalbuminuria with a median value of $125.6 \mathrm{mg} / \mathrm{g} ; 24 \mathrm{~h}$ of admission microalbuminuria persisted in $67 \%$; however, the median value had reduced to $62.6 \mathrm{mg} / \mathrm{g}$ [8]. Hence, the timing of sampling of urine to determine the ACR is crucial. With the onset of recovery, a rapid decline in ACR may be seen. Median ACR values were much higher in patients with sepsis and non-survivors [8].

Sachdev et al. studied the role of urinary ACR in children with varying severity of sepsis admitted to PICU. The study enrolled 138 participants, and serial ACR values were estimated. The ACR showed a rising trend with the increasing severity of sepsis. ACR values were greater in non-survivors. They concluded ACR $>102 \mathrm{mg} / \mathrm{g}$ correlated well with the lengthier duration of mechanical ventilation, necessity for inotropes, and death. ACR showed a good correlation with PELOD and PRISM scores [6].

In the present study, ACR showed a good correlation with PIM 2 score. The cutoff value of ACR derived to predict mortality was $110 \mathrm{mg} / \mathrm{g}$ which is comparable to previous studies $[6,12,13]$. In the present study, need for inotrope, presence of MODS, mortality, and duration of PICU stay were significant in ACR $>110 \mathrm{mg} / \mathrm{g}$ group.

A study by Thorevska in critically ill medical patients reported a prevalence of MA in $69 \%$ of the patients. $\mathrm{ACR} \geq 100 \mathrm{mg} / \mathrm{g}$ predicted mortality and hospital stay independently. Risk of death increased by 2.7 times when ACR was $>100 \mathrm{mg} / \mathrm{g}$ [13].

A study by Gosling et al. which recruited medical and surgical patients in adult ICU concluded that ACR predicted ICU mortality and inotrope requirement better than APACHE II and SOFA score [14]. Tayeh et al. reported that $\mathrm{ACR}$ at $24 \mathrm{~h}$ of admission was higher in patients who were 


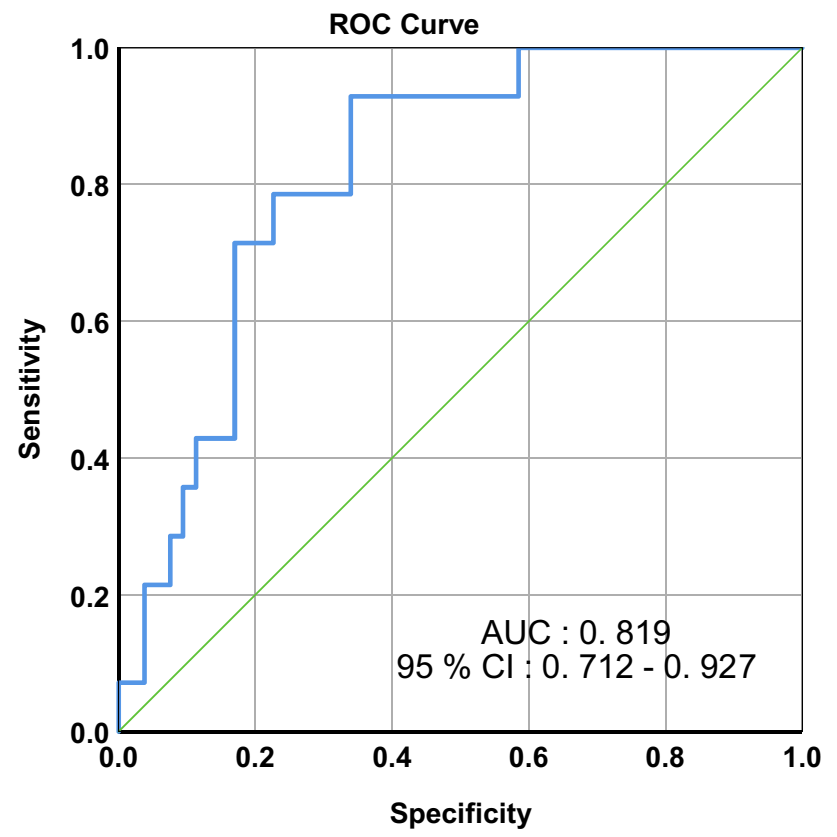

(a)

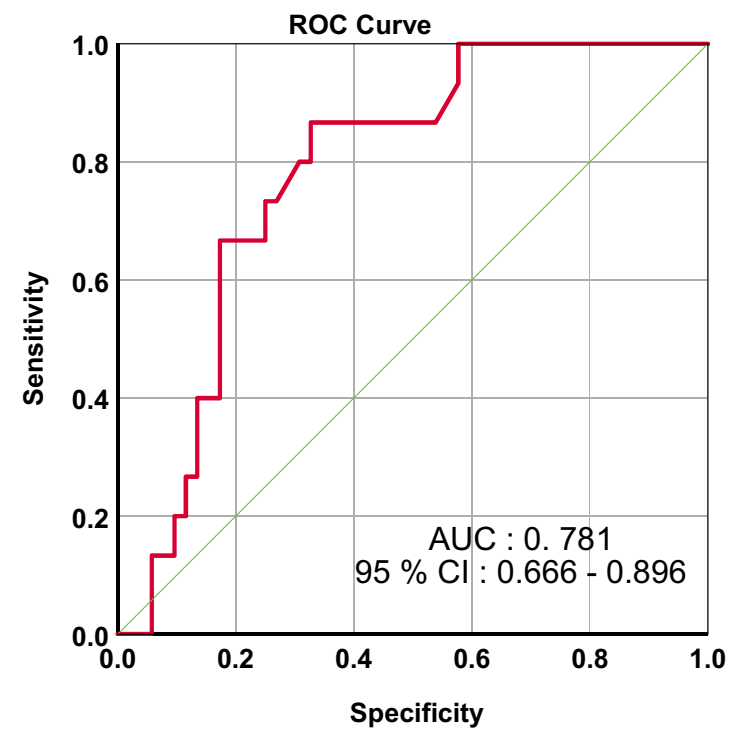

(b)

Fig. 1 Receiver operating characteristic curve of albumin creatinine ratio at admission for the need of inotrope (a) and multiple organ dysfunction syndrome $(\mathbf{b})$ in the pediatric intensive care unit

mechanically ventilated, those requiring inotropes. Increasing ACR was found to be a predictor of mortality [15].

\section{Limitations}

ACR was done only on admission. Repeating ACR subsequently and comparing it with patient recovery or worsening give a better idea of the predictive ability of ACR. The sample size of the present study was small. Studies with larger

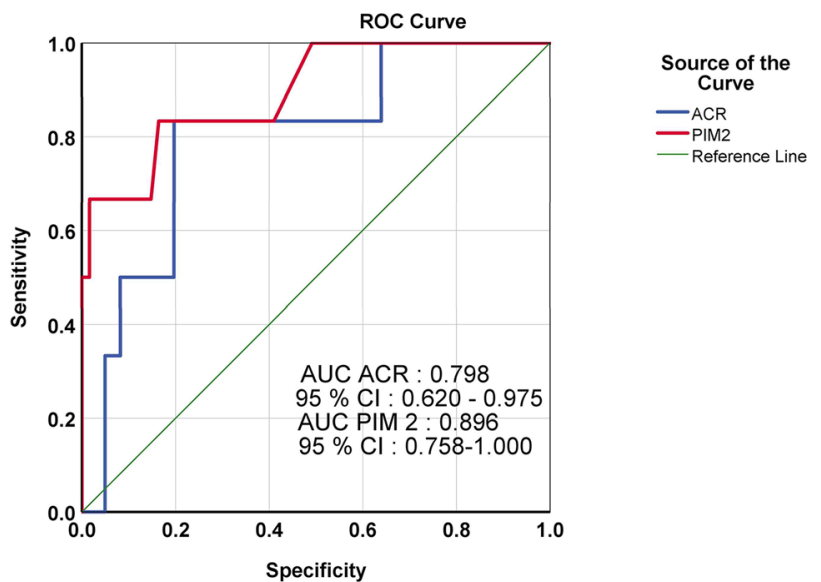

Fig. 2 Receiver operating characteristic curves of albumin creatinine ratio at admission and pediatric index of mortality 2 score for mortality in pediatric intensive care unit

Table 2 Comparison of the pediatric intensive care unit outcome parameters with albumin creatinine ratio $110 \mathrm{mg} / \mathrm{g}$ cutoff

\begin{tabular}{llll}
\hline Parameter & $\begin{array}{l}\text { ACR } \leq 110 \mathrm{mg} / \mathrm{g} \\
n=39 \\
n(\%)\end{array}$ & $\begin{array}{l}\text { ACR }>110 \mathrm{mg} / \mathrm{g} \\
n=28 \\
n(\%)\end{array}$ & $p$ value \\
& & & \\
\hline $\begin{array}{l}\text { Inotrope use } \\
\text { Present }\end{array}$ & $3(7.7)$ & $11(39.3)$ & 0.002 \\
$\quad$ Absent & $36(92.3)$ & $17(60.7)$ & \\
$\begin{array}{l}\text { Multiple organ } \\
\text { dysfunction syndrome }\end{array}$ & & & \\
$\quad \begin{array}{l}\text { Present } \\
\text { Absent }\end{array}$ & $3(7.7)$ & $12(42.9)$ & 0.001 \\
$\begin{array}{l}\text { Outcome } \\
\quad \text { Expired } \\
\text { Survived }\end{array}$ & $36(92.3)$ & $16(57.1)$ & \\
PICU stay days & $1(2.6)$ & $5(17.9)$ & 0.042 \\
$\quad$ Median (IQR) & $38(97.4)$ & $23(82.1)$ & \\
\hline
\end{tabular}

$A C R$ albumin creatinine ratio, $P I C U$ pediatric intensive care unit, $I Q R$ interquartile range

sample size help to generalize the results. For the prediction of PICU mortality, PIM 2 score was used instead of the updated version of PIM 3.

\section{Conclusion}

ACR was found to have significant relation to the need for inotropes, development of MODS, and prediction of mortality similar to PIM 2. In addition, ACR had a good correlation to the duration of the PICU stay. To conclude, ACR is an easy, cost-effective, and reliable test for predicting morbidity and mortality in a PICU setting. 
Funding Open access funding provided by Manipal Academy of Higher Education, Manipal.

\section{Declarations}

Ethical approval Approval was obtained from the ethics committee of Kasturba Medical College Mangalore. The procedures used in this study adhere to the tenets of the Declaration of Helsinki.

Informed consent Written informed consent was obtained from the parents.

Conflict of interest The authors declare no competing interests.

Open Access This article is licensed under a Creative Commons Attribution 4.0 International License, which permits use, sharing, adaptation, distribution and reproduction in any medium or format, as long as you give appropriate credit to the original author(s) and the source, provide a link to the Creative Commons licence, and indicate if changes were made. The images or other third party material in this article are included in the article's Creative Commons licence, unless indicated otherwise in a credit line to the material. If material is not included in the article's Creative Commons licence and your intended use is not permitted by statutory regulation or exceeds the permitted use, you will need to obtain permission directly from the copyright holder. To view a copy of this licence, visit http://creativecommons. org/licenses/by/4.0/.

\section{References}

1. Gulla KM, Sachdev A (2016) Illness severity and organ dysfunction scoring in pediatric intensive care unit. Indian J Crit Care Med 20:27-35

2. Gandhi J, Sangareddi S, Varadarajan P et al (2013) Pediatric index of mortality 2 score as an outcome predictor in pediatric intensive care unit in India. Indian J Crit Care Med 17:288-291

3. Slater A, Shann F (2004) The suitability of the pediatric index of mortality (PIM), PIM2, the pediatric risk of mortality (PRISM), and PRISM III for monitoring the quality of pediatric intensive care in Australia and New Zealand. Pediatr Crit Care Med $5: 447-454$
4. Slater A, Shann F, Pearson G (2003) PIM2: a revised version of the paediatric index of mortality. Intensive Care Med 29:278-285

5. Anil AB, Anil M, Yildiz M et al (2014) The importance of microalbuminuria in predicting patient outcome in a PICU. Pediatr Crit Care Med 15:e220-5

6. Sachdev A, Raheja K, Gupta N et al (2020) Association of urinary albumin: creatinine ratio with outcome of children with sepsis. Indian J Crit Care Med 24:465-472

7. Basu S, Chaudhuri S, Bhattacharyya M et al (2010) Microalbuminuria: an inexpensive, noninvasive bedside tool to predict outcome in critically ill patients. Indian J Clin Biochem 25:146-152

8. Basu S, Bhattacharya M, Chatterjee T et al (2010) Microalbuminuria: a novel biomarker of sepsis. Indian J Crit Care Med $14: 22-28$

9. Goldstein B, Giroir B, Randolph A (2005) International consensus conference on pediatric sepsis. International pediatric sepsis consensus conference: definitions for sepsis and organ dysfunction in pediatrics. Pediatr Crit Care Med 6:2-8

10. Societe Francaise d' Anesthesie et de Reanimation (2003) Scoring systems for ICU and surgical patients: PIM 2 (Paediatric Index of Mortality). https://sfar.org/scores2/pim22.php. Accessed 10 July 2021

11. Fleck A, Raines G, Hawker F et al (1985) Increased vascular permeability: a major cause of hypoalbuminemia in disease and injury. Lancet 1:781-784

12. Nismath S, Rao SS, Baliga BS et al (2020) Comparative validity of microalbuminuria versus clinical mortality scores to predict pediatric intensive care unit outcomes. Clin Exp Pediatr 63:20-24

13. Thorevska N, Sabahi R, Upadya A et al (2003) Microalbuminuria in critically ill medical patients: prevalence, predictors, and prognostic significance. Crit Care Med 31:1075-1081

14. Gosling P, Czyz J, Nightingale P et al (2006) Microalbuminuria in the intensive care unit: clinical correlates and association with outcomes in 431 patients. Crit Care Med 34:2158-2166

15. Tayeh O, Taema KM, Eldesouky MI et al (2016) Urinary albumin/ creatinine ratio as an early predictor of outcome in critically-ill septic patients. Egypt J Crit Care Med 4:47-55

Publisher's Note Springer Nature remains neutral with regard to jurisdictional claims in published maps and institutional affiliations. 